\title{
Molecular Pathways Engaged by Immunomodulatory Agents in Monoclonal Gammopathy-Associated Pure Red Cell Aplasia Rescue
}

\author{
Rakesh Verma* \\ Yale Cancer Center, Yale University, New Haven, CT, United States \\ Keywords: MGUS, multiple myeloma, IMiDs, immunomodulation, ikaros (IKZF1), CRBN
}

\section{INTRODUCTION}

OPEN ACCESS

Edited by:

Dimitar G. Efremov,

International Centre for Genetic

Engineering and Biotechnology, Italy

Reviewed by:

Giovanni D'Arena,

San Luca Hospital, Italy

Saad Zafar Usmani,

Levine Cancer Institute, United States Jérôme Paggetti,

Luxembourg Institute of

Health, Luxembourg

${ }^{*}$ Correspondence:

Rakesh Verma

rakesh.verma@aya.yale.edu

Specialty section:

This article was submitted to

Hematologic Malignancies,

a section of the journal

Frontiers in Oncology

Received: 03 April 2020

Accepted: 13 July 2020

Published: 27 August 2020

Citation:

Verma R (2020) Molecular Pathways

Engaged by Immunomodulatory

Agents in Monoclonal

Gammopathy-Associated Pure Red

Cell Aplasia Rescue.

Front. Oncol. 10:1490.

doi: 10.3389/fonc.2020.01490
Anemia remains a challenge for most cancer patients treated with therapies that include chemotherapy to current immunotherapy or chemo-immunotherapy treatments. Multiple myeloma (MM) is a plasma cell neoplasm marked by the clonal proliferation of malignant plasma cells. Preceding stage in MM includes asymptomatic stages including monoclonal gammopathy of undetermined significance or MGUS before the disease progression to clinical MM. One of the clinical features of $\mathrm{MM}$ at diagnosis includes anemia, presenting in a majority of MM patients. Pathological changes in bone marrow microenvironment in monoclonal gammopathy/MGUS and MM contribute to the pathological imbalance in erythroid cell production, leading to lowered downstream mature erythroid cells. Molecular events and pathways that contribute to this pathological remodeling of erythroid pathway-elements remain unclear and need further investigations.

\section{ANEMIA AND MYELOMA PROGRESSION}

Anemia is among the key clinical hallmarks of myeloma diagnosis and also requires clinical management in patients with uncontrolled active progressive disease (1). Presentation of clinical myeloma involves preceding asymptomatic stages including monoclonal gammopathy of undetermined significance. Data on monoclonal gammopathy or MGUS associated pure red cell aplasia (PRCA) has remained obscure and requires more attention beyond a few recent reports (2). Pure red cell aplasia is marked by absence of precursor erythroid cells or erythroblasts, eventually leading to erythroid hypoplasia in the bone marrow of these patients. Novel targets including Regulator of Hemoglobinization and Erythroid cell eXpansion or RHEX in the erythropoietin $(\mathrm{EPO}) / \mathrm{EPO}$ receptor (EPOR) pathway that play key roles in erythroid cell maturation and development exclusively in humans were recently reported (3). Molecular mechanisms underlying the development of PRCA remain largely unidentified with description of immune-mediated either humoral or cellular ablation of erythroid precursors in the bone marrow. This translates into inefficient erythropoiesis leading to requirement of frequent blood transfusions to rescue anemia in these MGUS and MM patients.

Immunomodulatory (IMiD) agents like pomalidomide have recently been reported to induce increased in vivo $\gamma$-globin levels in erythrocytes of multiple myeloma patients (4). These studies have revealed the molecular mechanisms by which IMiDs like pomalidomide and lenalidomide reactivate fetal hemoglobin in myeloma patients. These pathways further help translate this 
activation of fetal hemoglobin into recovery from anemia clinically, while still maintaining the anti-myeloma effects of this class of $\mathrm{IMiD}^{\mathrm{R}}$ drugs. Although the molecular mechanisms underlying the anti-myeloma activity of $\mathrm{IMiDs}^{\mathrm{R}}$ have been reported to involve the Cullin 4A (CUL4A)- Cereblon (CRBN) E3 ligase complex mediated proteasomal degradation of downstream targets (5). These CRBN targets include transcription factors like IKAROS (IKZF1), while additional molecular pathways underlying recovery from anemia in myeloma remain IKAROS or IKZF1 independent (4-6). Specifically, transcriptional modules affected by pomalidomide or lenalidomide has been reported to involve $\gamma$-globin repression included BCL11A, SOX6, IKZF1, KLF1, and LSD1. But IKAROS (IKZF1) was not identified as the key effector of this program, as IKZF1 ablation was not sufficient to phenocopy pomalidomide treatment (4). These findings point to the existence of additional pathways that may include EPO/EPOR dependent mechanisms driving the onset and recovery from anemia in MGUS and MM patients (Figure 1).

It is to be noted that monoclonal gammopathy-associated PRCA might be a paraprotein-related phenomenon. It can be further speculated that there could be a functional relationship between altered plasma cell and erythroid precursor, with a possible causal relationship between $\mathrm{M}$-protein response and hematological response including PRCA reversal in the
PRCA bone marrow after treatment with pomalidomide or IMiDs (2). A number of intriguing questions regarding the underlying molecular and pathogenetic mechanisms still remain unanswered. Modulation of tumor microenvironment is aptly speculated via possible roles of IgG monoclonal protein for erythroid inhibition. But based on the roles of IKZF1 and CRBN for eliciting anti-myeloma effects for any possible hematological response accompanying reduction in the disease burden (Mprotein), it remains unclear if recovery of erythroid progenitors is CRBN or IKZF1 independent. Additionally, possible changes in the $\gamma$-globin levels can be further speculated in patients with hematological recovery after anti-myeloma therapies $\left(\mathrm{IMiDs}^{\mathrm{R}}\right.$ based) as reported by Dulmovits et al. (4).

\section{DISCUSSION}

Detailed molecular mechanisms describing the additional targets of IMiD action remain an active area of research (7) and these additional pathways may contribute to the recovery of erythroid elements in the bone marrow of MM patients. This is further validated by the data on absence of expected JAK2 V617F and CALR Type 1/2 type mutations in the monoclonal gammopathy-associated PRCA cohort reported by Korde et al. (2) eliminating the dysfunctional EPOR mediated JAK2-STAT5 pathway circuitry.

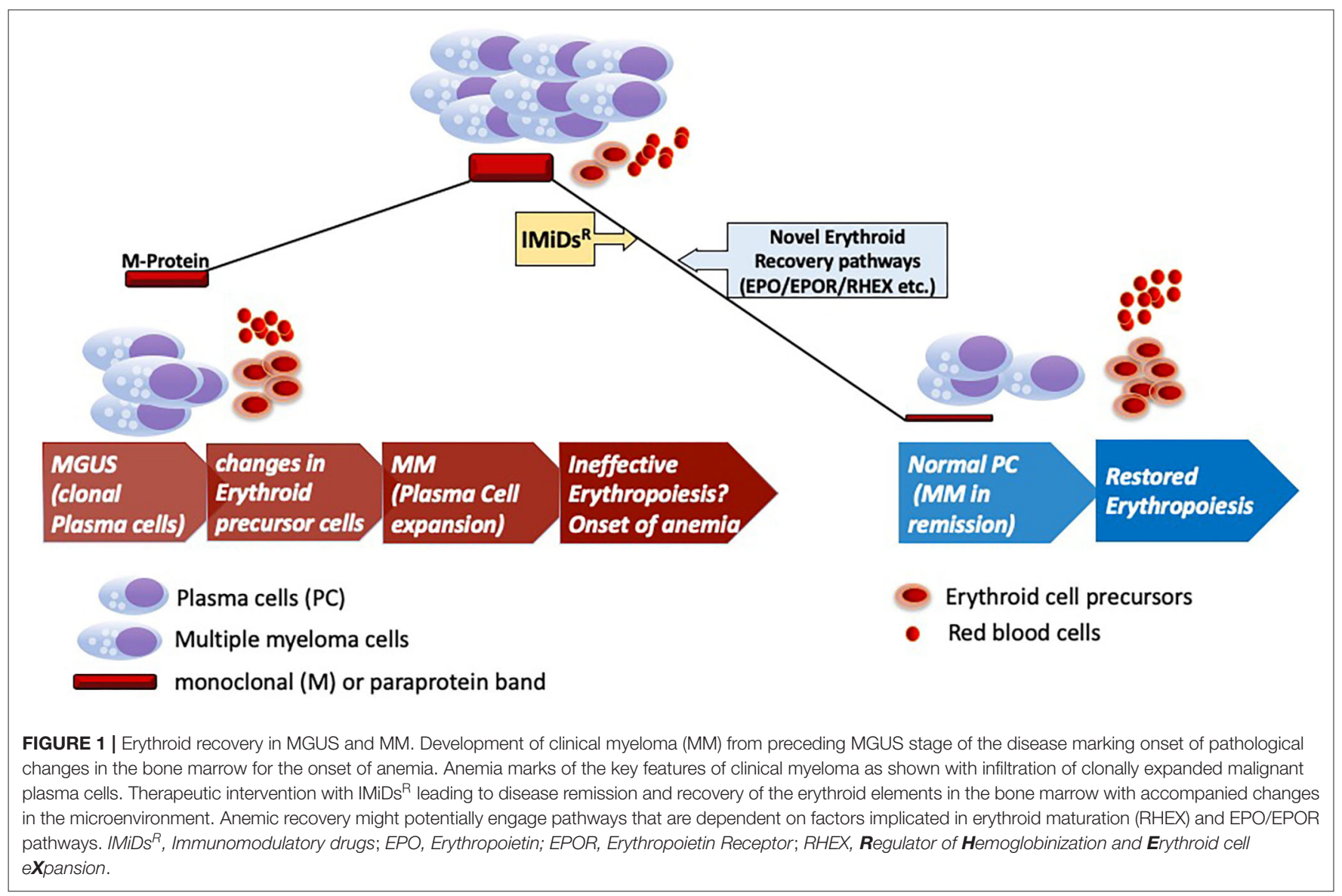


Monoclonal gammopathy-associated PRCA illustrates an open ended clinical challenge to describe the underlying possible pathogenetic mechanisms by future mechanistic studies (Figure 1). Anti-myeloma drugs like $\mathrm{IMiDs}^{\mathrm{R}}$ drive the erythroid recovery in this pathology but independent of currently known molecular pathways that exclusively drive the action of these drugs on myeloma cells and potentially some immune cell subsets. Absence of RTK mutations further makes a strong case for an alternative mechanistic active in the progenitor cells under the action of novel agents like pomalidomide or lenalidomide for erythroid recovery in MM. Recent discovery of novel regulators like RHEX implicated in the human erythroid cell maturation combined with the findings of Dulmovits et al. proposes further investigations of erythroid maturation pathways in MM and Monoclonal gammopathy or MGUS associated PRCA to uncover the underlying molecular mechanism. With the progress of cell therapies like the BCMA targeting CAR-T cells for late stage or relapsed/refractory MM (8), these insights

\section{REFERENCES}

1. Palumbo A, Anderson K. Multiple myeloma. N Engl J Med. (2011) 364:104660. doi: 10.1056/NEJMra1011442

2. Korde N, Zhang Y, Loeliger K, Poon A, Simakova O, Zingone A, et al. Monoclonal gammopathy-associated pure red cell aplasia. $\mathrm{Br}$ J Haematol. (2016) 173:876-83. doi: 10.1111/bjh.14012

3. Verma R, Su S, McCrann DJ, Green JM, Leu K, Young PR, et al. RHEX, a novel regulator of human erythroid progenitor cell expansion and erythroblast development. J Exp Med. (2014) 211:1715-22. doi: 10.1084/jem.20130624

4. Dulmovits BM, Appiah-Kubi AO, Papoin J, Hale J, He M, Al-Abed Y, et al. Pomalidomide reverses gamma-globin silencing through the transcriptional reprogramming of adult hematopoietic progenitors. Blood. (2016) 127:148192. doi: 10.1182/blood-2015-09-667923

5. Kronke J, Udeshi ND, Narla A, Grauman P, Hurst SN, McConkey M, et al. Lenalidomide causes selective degradation of IKZF1 and IKZF3 in multiple myeloma cells. Science. (2014) 343:301-5. doi: 10.1126/science.1244851

6. Lu G, Middleton RE, Sun H, Naniong M, Ott CJ, Mitsiades CS, et al. The myeloma drug lenalidomide promotes the cereblon-dependent destruction of Ikaros proteins. Science. (2014) 343:305-9. doi: 10.1126/science.1244917 into anemic recovery would benefit the ultimate advancement of advanced CAR-T based therapies for early stages of MM. Future detailed mechanistic studies can help identify alternative treatment strategies as compared to blood transfusions for these MGUS/MM patients for efficient anemic recovery.

\section{AUTHOR CONTRIBUTIONS}

RV prepared and wrote the manuscript.

\section{ACKNOWLEDGMENTS}

RV would like to extend sincere thanks to the staff at Yale Cancer Center, Section of Hematology and Yale Immunobiology for their kind support. RV would like to acknowledge Prof. Daniel DiMaio, MD, PhD (Genetics) and Prof. Richard Flavell, PhD, FRS (Immunobiology) at Yale University School of Medicine for their support.

7. Verma R, Mai Z, Xu M, Zhang L, Dhodapkar K, Dhodapkar MV. Identification of a cereblon-independent protein degradation pathway in residual myeloma cells treated with immunomodulatory drugs. Blood. (2015) 126:913. doi: 10.1182/blood.V126.23. 913.913

8. Raje N, Berdeja J, Lin Y, Siegel D, Jagannath S, Madduri D, et al. AntiBCMA CAR T-cell therapy bb2121 in relapsed or refractory multiple myeloma. N Engl J Med. (2019) 380:1726-37. doi: 10.1056/NEJMoa 1817226

Conflict of Interest: The author declares that the research was conducted in the absence of any commercial or financial relationships that could be construed as a potential conflict of interest.

Copyright (c) 2020 Verma. This is an open-access article distributed under the terms of the Creative Commons Attribution License (CC BY). The use, distribution or reproduction in other forums is permitted, provided the original author(s) and the copyright owner(s) are credited and that the original publication in this journal is cited, in accordance with accepted academic practice. No use, distribution or reproduction is permitted which does not comply with these terms. 